\title{
Monitoreo y evaluación de políticas públicas en América Latina: brechas por cerrar.
}

Monitoring and Evaluation of Public Policies in Latin America: Bridging the Gaps.

María Angélica Pignatta Universidad Nacional de Rosario (UNR)

Facultad Latinoamericana de Ciencias Sociales (FLACSO) Argentina. angelica.pignatta@gmail.com
Fecha de recepción:

4.11.14

Fecha de aceptación: 12.2.15

\section{Resumen}

La convicción sobre la importancia de monitorear y evaluar las políticas públicas se ha instalado paulatinamente en la región latinoamericana; sin embargo, aún debe enfrentar importantes retos tanto en el campo conceptual y en las perspectivas predominantes como en la práctica de la gestión pública. En este escenario, el trabajo se centra en profundizar sobre la dupla monitoreo y evaluación, primero, para clarificar las distinciones y relaciones entre ambos. Segundo, se abordan ciertos desafios para la gestión pública: acortar las brechas de capacidades institucionales para fomentar su práctica desde la incorporación del enfoque de derechos y modificar las perspectivas que priman en la administración pública priorizando el aprendizaje de forma que favorezcan la utilización de los hallazgos y recomendaciones.

Palabras clave: monitoreo y evaluación, políticas públicas, capacidades institucionales, brechas de capacidad, América Latina

\section{Abstract}

The belief in the importance of monitoring and evaluation of public policy has gradually established in Latin America; however, it still faces major challenges not only in the conceptual field and the predominant perspectives, 
but also, in public management practice. In this scenario, the paper focuses on monitoring and evaluation (MEE). First, to clarify the distinctions and relationships between them and, second, to address certain challenges in public management: on the one hand, bridging institutional capacities gaps to promote their practice from a right-based approach and, on the other hand, changing prevailing perspectives in public management/government by prioritizing learning to promote the use of findings and recommendations.

Key Words: monitoring and evaluation - public policystate capabilities - capability gaps- Latin America

\section{Introducción}

La preocupación por contar con sistemas destinados a monitorear y evaluar la gestión de políticas sociales está presente desde hace más de dos décadas; sin embargo, en la mayoría de los países latinoamericanos sigue siendo una meta por alcanzar (Cecchini y Martínez 2011).Aún se identifican amplias brechas en los niveles de institucionalización del monitoreo y la evaluación entre los diversos países latinoamericanos y en sus distintos niveles de gobierno. Los desafios para la región se centran tanto en institucionalizar su práctica para el perfeccionamiento de la calidad y para la gestión de políticas como en modificar las perspectivas de la evaluación y el monitoreo que priman en la administración pública, priorizando el aprendizaje de forma que favorezcan la utilización de los hallazgos y recomendaciones.

En este marco, se entiende a las políticas públicas como un proceso, "como secuencia de hechos y decisiones que implican un cierto avance o modificación de la realidad" (Subirats 1989:41). El foco se coloca en entender a la política pública como una hipótesis, susceptible de error, sobre todo al considerar que los problemas públicos son escabrosos, pluridimensionales y cambiantes y que en su atención concurren un gran número de actores (Aguilar Villanueva 1992). En este sentido, cobra relevancia colocar el énfasis en la identificación de aspectos de mejora de las políticas públicas y en la necesidad de someterlas a una examinación permanente para valorar en qué medida su desempeño y sus resultados implican una transformación positiva de la realidad.

En el presente trabajo se pretende profundizar sobre la dupla monitoreo y evaluación (MyE) - también denominada seguimiento y evaluación (SyE) - haciendo énfasis en tres ejes de análisis. ${ }^{1}$ Primero, cuáles son las distinciones conceptuales entre ambos com- 
ponentes de esta dupla y las relaciones que pueden establecerse entre ellos. Segundo, se abordan ciertos desafíos de los sistemas de monitoreo y evaluación de la región, y su vinculación con los marcos y perspectivas subyacentes, para convertirse en una clave que se oriente al mejoramiento de las políticas públicas. Finalmente, se argumenta la necesidad de modificar las visiones predominantes en torno al monitoreo/seguimiento y evaluación en América Latina para revalorizar la función de aprendizaje.

\section{Monitoreo, Seguimiento y Evaluación: una aproximación conceptual a las distinciones y vinculaciones}

Resulta relevante considerar cuáles son las distinciones que habitualmente se han realizado entre monitoreo, seguimiento y evaluación, qué perspectivas predominantes subyacen bajo estas distinciones y de qué manera influyen en las conexiones y desconexiones entre la dupla MyE.

Una amplia bibliografia se ha ocupado de conceptualizar las nociones de monitoreo, seguimiento y evaluación. Sin embargo, no resulta extraño que estos términos continúen siendo utilizados, tanto en el campo académico como en el de la gestión pública, ya de manera indistinta o, al contrario, poniendo énfasis en sus aspectos distintivos más que en sus vinculaciones o relaciones. Se propone en este trabajo delinear un marco conceptual acorde a un punto medio que pueda diferenciar aspectos claves y a la vez reconocer sus conexiones. De esta manera, cabe plantear como interrogantes: ¿Cuáles son los elementos distintivos que caracterizan a cada uno de los componentes de la dupla? ¿Qué aspectos distintivos pueden ser objeto de debate? ¿Cómo se configura la relación entre ambos componentes?

Una de las definiciones más difundidas entre los autores de la región ha sido la acuñada en el glosario de la Organización para la Cooperación y el Desarrollo Económico (OECD). ${ }^{2}$ Esta define el seguimiento o monitoring como una función continua que utiliza la recopilación sistemática de los datos aportados por los indicadores predefinidos para proporcionar información al conjunto de actores interesados acerca de la utilización de los fondos asignados, de avance y del logro de los objetivos (OECD; 2002). Por otra parte, conceptualiza a la evaluación como la "apreciación sistemática y objetiva de un proyecto, programa o política en curso o concluido, de su diseño, su puesta en práctica y sus resultados. El objetivo es determinar la pertinencia y el logro de los objetivos, así como la eficiencia, la eficacia, el impacto y la sostenibilidad para el desarrollo" (OECD; 2002: 21 - 22).

En esta conceptualización se pueden observar dos aspectos significativos. Por un lado, el concepto de seguimiento es considerado como sinónimo a la noción inglesa de monitoring. En Latinoamérica el término "seguimiento" ha sido adoptado como una categoría más próxima en tanto "monitoreo" es considerado un anglicismo, aunque esto se basa 
en el desconocimiento de su origen latino y en limitarlo al significado anglosajón de origen del término. Si bien la noción monitoreo no aparece a priori como tal en el diccionario de la lengua de la Real Academia Española (2012), se encuentra en cambio el término "monitor/a"3 que reconoce significados tanto de origen anglosajón asociados a la idea de control, como de origen latín que incluye además otros sentidos, como la persona que guía el aprendizaje y diversas acepciones que pueden vincularse al uso de la información documentada para la toma de decisiones o para la rendición de cuentas, al acompañamiento y al recordatorio durante la propia marcha de la acción. ${ }^{4}$

El segundo aspecto que se desprende de la definición de la OECD hace referencia a las distinciones planteadas entre la dupla monitoreo/seguimiento y evaluación. En primer lugar, el monitoreo o seguimiento se destaca como una función continua, lo que puede considerarse como un aspecto distintivo destacado por diversos autores que lo han caracterizado como un proceso más regular, continuo, recurrente y/o permanente (Guzmán 2007; Cunill y Ospina 2007; PNUD 2009; Di Virgilio y Solano 2013; Pallavecini 2014). En contraste, se plantea que la evaluación se realiza en períodos o momentos establecidos y es de carácter puntual sobre una intervención (Cunill y Ospina 2007; Di Virgilio y Solano 2013; Pallavecini 2014). En relación a esto, se establece que mientras el monitoreo o seguimiento se realiza paralelamente a la implementación o ejecución de los planes (Pallavecini 2014); la evaluación puede realizarse sobre un programa en curso o concluido y, en este marco, los tipos de evaluación pueden diferenciarse de acuerdo al momento en que ésta se realiza. La evaluación ex-ante (de pre-decisión, de factibilidad o de pertinencia) hace referencia a aquella que se realiza antes de iniciar un programa o proyecto para tomar una decisión relativa a si debe implementarse o no. Este tipo de evaluación hace foco en la formulación al establecer la pertinencia, coherencia interna, factibilidad y viabilidad de las acciones planteadas en el documento de formulación del proyecto (Niremberg, Brawerman y Ruiz 2003). Segundo, la evaluación ex-post - que puede incluir de fin, de proyecto, a posteriori, terminal, de resultados, de impacto - es la que se realiza una vez concluida la ejecución del proyecto y pone énfasis en los resultados obtenidos para valorar en qué medida se alcanzaron los objetivos previstos y cuáles han sido los efectos buscados y no buscados, atribuibles al proyecto (Niremberg et al 2003). Finalmente, la evaluación concomitante -o de progreso, proceso, gestión, concurrente, intermedia- es aquella que se realiza durante la implementación de la política y se orienta a evaluar en qué medida ésta se va cumpliendo de acuerdo con la propuesta inicial. Así coloca el foco en los procesos, en el modo en que se desarrollan las actividades previstas (Niremberg et al 2003) y busca proporcionar elementos para la toma de decisiones que permiten reforzar o reorientar diversos aspectos para alcanzar resultados favorables.

Algunos autores ligan la evaluación concomitante y el monitoreo o seguimiento. Karen Mokate (2003) utiliza como sinónimos, por un lado, el monitoreo y la evaluación de 
procesos y, por el otro, la evaluación propiamente dicha y la evaluación de objetivos. Si bien esta distinción puede llevar a la confusión de considerar que el monitoreo o seguimiento no incluye o aborda cuestiones relativas a los objetivos, la autora referida destaca la complementariedad de ambos componentes en un proceso integrado y continuo. En un sentido similar, Vinocur y Ruiz (2013) proponen la inexistencia de una diferenciaicón entre la evaluación de procesos y el monitoreo, ya que éste último constituye una típica actividad de evaluación interna. Más allá de las divergencias que pueden establecerse entre evaluación de procesos y monitoreo o seguimiento, es posible afirmar que una especificidad del monitoreo y/o seguimiento es que se realiza durante la implementación de una política.

Otro aspecto que habitualmente es señalado como diferencia central entre el monitoreo/seguimiento y la evaluación refiere a que el primero se asocia a una aplicación predominantemente interna propia de las organizaciones, mientras que la evaluación puede ser clasificada en diversos tipos de acuerdo a quien realiza la misma (externa, mixta, interna, participativa o autoevaluación). Por otro lado, se identifica en diversas conceptualizaciones que la noción de evaluación es caracterizada como una apreciación "objetiva" y de forma independiente asociada generalmente al tipo de evaluación externa. Según el PNUD, el aspecto distintivo clave entre el seguimiento y la evaluación refiere a dos aspectos. Por un lado, las evaluaciones son más rigurosas en sus procedimientos, diseño y metodología y generalmente implican un análisis más amplio (PNUD 2007). De acuerdo con esto, se ha planteado que el seguimiento no explica las causas de lo que se documenta, ni ofrece la oportunidad de aprender la complejidad de los procesos (Cunill y Ospina 2007). Por otro lado, se plantea que las evaluaciones - a diferencia del seguimiento - son realizadas de forma independiente para proporcionar una valoración objetiva.

En este punto cabe considerar algunos interrogantes que derivan de esta distinción y abren el debate: ¿Es la mirada externa más adecuada que la interna? ¿En qué medida puede garantizarse y es deseable la neutralidad? Algunos autores sostienen que la mirada externa de la evaluación le otorga mayor legitimidad a las conclusiones en tanto permite una valoración objetiva y desprovista de intereses internos. En esta línea, se ha planteado que el tipo de evaluación óptima es la externa ya que es objetiva: "los actores que la realizan no tienen un interés interno, son neutrales y, por lo tanto, sus resultados son transparentes y lo más cercanos a la realidad” (Romero Jiménez, 2013:37). Desde esta perspectiva, el monitoreo o seguimiento tiende a ser devaluado como una actividad potencialmente "contaminada" por las visiones internas.

En el presente trabajo se argumenta que aunque la mirada externa, en ocasiones, puede aportar un valor adicional, es discutible tanto su asociación directa a un carácter independiente como así también su pretensión de neutralidad y, por sobre todo, que sea 
siempre la forma más adecuada de introducir mejoras. Una advertencia interesante refiere a considerar que las evaluaciones independientes no son equivalentes a las evaluaciones externas (ni las evaluaciones internas y evaluaciones no independientes) ya que la independencia de las evaluaciones no depende de que sean externas y, por otra parte, las evaluaciones internas pueden ser independientes (Feinstein 2010). A la vez, se considera que aun cuando se reconoce que la mirada desde afuera de la escena permite aportar otras perspectivas, esto no quiere decir que el evaluador tenga una visión neutra sino, al contrario, su posicionamiento puede ser justamente el valor agregado. En este marco, se hace necesario reconocer la subjetividad intrínseca del monitor/evaluador y su "carga ideológica" siendo menester hacerse cargo de la misma y explicitarla para garantizar el rigor científico. Cabe aclarar que la rigurosidad científica de una investigación no se vincula solo con la búsqueda de "racionalidad" sino con la calidad de las decisiones que los investigadores hacen a lo largo de todo el proceso de investigación, que incluye los métodos de recolección de información; la definición del problema, el marco conceptual, la generación de hipótesis, el trabajo de campo y el análisis e interpretación de los resultados (Petersen, 1992). Además, en la práctica, la visión externa puede encontrar dificultades para captar la complejidad de lo social al desconocer la pluralidad de sentidos y de actores que intervienen en una política. Asimismo, es posible encontrar resistencias por parte de los propios implementadores, lo que se vincula estrechamente con las perspectivas subyacentes que asocian la evaluación al control y generan un clima de aversión ante la misma. En este sentido, resulta interesante considerar la importancia de la complementariedad entre las evaluaciones internas (o las autoevaluaciones) y el seguimiento o monitoreo con las evaluaciones externas. Siguiendo a Feinstein (2010), se considera que el desdén por la valoración interna ha afectado negativamente la práctica de la evaluación, ya que no permite contar con información clave para la realización de las evaluaciones; en cambio, una buena práctica que se ha ido imponiendo es la de complementar las autoevaluaciones - podemos agregar y el monitoreo o seguimiento con evaluaciones externas.

Al priorizar la búsqueda de neutralidad y objetividad, el monitoreo y la evaluación se asocian a un proceso plenamente racional; cuando en realidad éstos están atravesados por decisiones políticas en términos de definición de prioridades sociales, distribución de recursos e incluso de poder. Diversos estudios han coincidido en afirmar que el monitoreo y la evaluación no pueden ser entendidos como rituales neutros o vacíos, sino que el juicio de valor tiene un componente claramente político (Di Virgilio y Solano; 2013). En esta línea, se plantea la evaluación como una cuestión política - no sólo técnica- "que concierne tanto a gobernantes como a gobernados" (Ospina 2000: 21). Otro elemento distintivo que se hace necesario debatir refiere a la consideración de que lo propio de la evaluación - y diferencia esencial con el monitoreo - es que incluye un juicio de valor. Si bien es cierto que la propia definición de evaluación enfatiza la idea de valoración o estimación, plantear esto como una distinción esencial y absoluta en 
torno al monitoreo o seguimiento, favorece una concepción restringida del mismo. Al excluir de su órbita la emisión de valoraciones, éste corre el riesgo de quedar desprovisto de un sentido profundo y, en cambio, asociarse a una acción casi autómata en la cual el foco se coloca en la cuestión técnica de cumplimiento de ciertos requisitos en términos de metas e indicadores; más que en su utilización en procesos de retroalimentación, la introducción de mejoras, la toma de decisiones, la definición de prioridades y la generación de cambios. De acuerdo con este argumento, el seguimiento o monitoreo es definido también por Cunill y Ospina (2007) como la valoración recurrente de avance de una intervención, usando indicadores de desempeño seleccionados a priori.

Asimismo, cabe considerar qué tipo de indicadores incluye el monitoreo o seguimiento y cuáles la evaluación. ${ }^{5}$ Algunos autores sostienen que el seguimiento se centra principalmente en indicadores de proceso y producto (Guzman 2007). En cambio, desde el enfoque centrado en la gestión basada en resultados $(\mathrm{GbR}),{ }^{6}$ los sistemas de monitoreo incorporan indicadores no sólo de insumos, actividades y productos sino también de resultados o vinculados a los cambios sociales que la intervención estatal debe producir. En esta línea, según Cunill y Ospina (2007), los indicadores de seguimiento pueden ser cualitativos o cuantitativos, y pueden enfocar el proceso y/o los resultados. Igualmente, la concepción del PNUD sostiene que el seguimiento "no sólo se preocupa por la cuestión de si estamos emprendiendo las acciones que dijimos que haríamos, sino que también pregunta si estamos avanzando para lograr los resultados que dijimos que queríamos alcanzar" (PNUD; 2009: 8).

Finalmente, al analizar las distinciones planteadas por diversos autores cabe preguntarse si hay una diferencia en las funciones o el para qué de cada uno de los elementos de la dupla MyE. De acuerdo al análisis de las distinciones entre ambos conceptos, se puede detectar que el seguimiento o monitoreo enfatiza en generar información que pueda realizar ajustes a lo planificado, proporcionar información de avance y a la vez sobre el logro de los objetivos o los resultados que se propusieron alcanzar (PNUD 2009; OECD 2002). La evaluación se realiza también para establecer en qué medida se están logrando los objetivos estipulados como así también para determinar su pertinencia y encontrar factores explicativos a fin no sólo de documentar patrones, sino también de explicarlos y/o comprenderlos, capturando su complejidad (PNUD 2009; Cunill y Ospina 2007).

Lo que se quiere enfatizar, más allá de estas distinciones, refiere a las relaciones que pueden darse entre ambos componentes. Tal como se describió, las diferencias se vinculan más bien a una cuestión de énfasis en lo que hace uno y lo que hace el otro. En este sentido, la dupla se entiende como un continuo cuyas relaciones tiende a ser de carácter complementario e interdependientes. Se puede reconocer que el monitoreo o seguimiento encuentra un valor instrumental para la evaluación en tanto también puede 
contribuir a la evaluabilidad de políticas públicas, programas y proyectos. La evaluabilidad hace referencia a la "medida en que puede evaluarse una actividad o programa de manera fiable y creíble" (OECD 2002: 21). La generación de información sistematizada que aporta el monitoreo o seguimiento constituye un insumo para la evaluación. Pero cuando éste no se realiza, la evaluación termina por suplirlo y, de esta manera, sale a cumplir funciones que ya podrían estar adelantadas por el monitoreo. A la vez, el seguimiento es necesario y facilita la evaluación, pero la evaluación además utiliza la recopilación de nuevos datos adicionales y diferentes marcos para el análisis (PNUD 2009). A la inversa, la evaluación también es un insumo para el monitoreo en tanto éste debe darle seguimiento a las recomendaciones producto de la evaluación para buscar que sean incorporadas y puedan repercutir en procesos de mejoras. Los cambios que se generen producto de la evaluación pueden significar modificaciones adicionales en la recolección de datos para el seguimiento (PNUD 2009).

En este sentido, se plantea una relación simbiótica entre ambos elementos que se vislumbra, además, en cómo el desarrollo de uno conlleva al otro y viceversa. Para ilustrar esta relación, cabe considerar los casos de México y Colombia, dos países que se caracterizan por su alto grado de institucionalización del monitoreo y evaluación en relación a los países de la región latinoamericana. ${ }^{7}$ Lo relevante que muestran ambos casos para ejemplificar esta relación es que - aún por trayectorias diversas - el fortalecimiento de un componente de la dupla ha llevado al desarrollo del otro y viceversa. Mientras en Colombia, el Sistema Nacional de Evaluación de Gestión y Resultados (SINERGIA) priorizó el seguimiento desde el 2000 y a partir del año 2010 se fortalece el componente de evaluación; en la experiencia de México - principalmente en el Consejo Nacional de Evaluación (CONEVAL) - se priorizó la evaluación externa y en la actualidad hay una tendencia con énfasis predominante en el trabajo interno de las áreas. Por otra parte, en algunos países latinoamericanos con un nivel medio de institucionalización de sus sistemas de monitoreo y evaluación se han focalizado los esfuerzos en un solo componente de la dupla: Argentina, Uruguay y Costa Rica en, a pesar de sus denominaciones, los llamados sistemas de seguimiento y evaluación nacional son en rigor sistemas de seguimiento o monitoreo (Feinstein 2012; Zaltman 2006). En el marco de la consolidación de los procesos de institucionalización de los sistemas de estos países, se hace necesario avanzar en la complementariedad con el componente de evaluación a partir de sus relaciones de reforzamiento mutuo y, de esta manera, en el mismo proceso fortalecer el componente de seguimiento o monitoreo.

Ante el argumento presentado, se resiste la tentación de esbozar un cuadro comparativo que sintetice las diferencias planteadas entre monitoreo/seguimiento y evaluación para, en cambio, subrayar en esta oportunidad sus vinculaciones como complementarias y analizar de qué manera se pueden acortar las brechas que se plantean, tanto a nivel teórico como en la práctica, para poder pensar en sistemas que verdaderamente incluyan 
conjuntamente el monitoreo y la evaluación. En la sección siguiente se coloca el foco en el análisis de los sistemas de $\mathrm{MyE}$ (o SyE), como un proceso continuo y, en función de esto, se identifican algunos de sus principales retos - en términos de brechas por cerrar y perspectivas de cambio- en el contexto de la región latinoamericana

\section{Retos de los sistemas de monitoreo y evaluación en América Latina}

A continuación se identifican ciertos rasgos de los sistemas de monitoreo y evaluación en la región que constituyen desafíos vinculados con perspectivas o enfoques que pueden constituir una clave para el mejoramiento de las políticas públicas: las extensas brechas de institucionalidad en términos de capacidades entre países, sectores y niveles de gobierno y su vinculación con la incorporación de una perspectiva de derechos, y la utilización de los hallazgos o recomendaciones de las evaluaciones explorando su vinculación con la función de aprendizaje. Para ilustrar, se tomarán las experiencias de algunos países latinoamericanos.

\subsection{Brechas de capacidad institucional y el enfoque de derechos.}

La institucionalización del monitoreo y la evaluación de políticas públicas en América Latina ha ido avanzando paulatinamente a finales del siglo XX y se ha acelerado a principios del siglo XXI (Feinstein; 2012). Sin embargo, los niveles de institucionalidad de los sistemas MyE encuentran importantes brechas entre los países, sectores y niveles de gobierno.

Las experiencias de Colombia y México pueden reseñarse por su alto nivel de institucionalización en comparación al resto de la región. En el primer caso, resulta relevante que la propia Constitución de 1991 introduce la obligatoriedad de evaluar las políticas públicas por parte de la entidad nacional de planeación y de los mismos organismos departamentales de planeación. En la experiencia mexicana destaca la aprobación en el año 2000 de una ley que requiere una evaluación anual de todos los programas administrados por el poder ejecutivo federal y la Ley General de Desarrollo Social del 2004 que institucionalizó el proceso de evaluación e instituyó el CONEVAL (Arizti y Fernando Castro 2010). Sin embargo, es notorio que la institucionalidad no se limita sólo al marco legal-normativo sino que hace referencia a las reglas de juego formales e informales, a los marcos legales y culturales.

En este sentido, Arizti y Fernando Castro (2010) detectan una brecha institucional al analizar el caso de México ya que, aunque se cuenta con un marco legal para la evaluación y el CONEVAL pueda considerarse una institución con un diseño apropiado para la evaluación de programas y políticas de desarrollo social, el monitoreo y la evaluación del sector público no están completamente institucionalizadas en el nivel central, donde se evidencian fuertes brechas entre los sectores (con mayor desarrollo institucional en los sectores de desarrollo social y de educación) y en los gobiernos subnacionales. Al 
igual que en el resto de los países de la región, el reto se focaliza en institucionalizar la práctica del monitoreo y la evaluación al interior de la administración pública como un instrumento reconocido para el perfeccionamiento de la calidad y para la gestión de políticas.

Tanto en los casos planteados de México y Colombia como en otros países de la región con desarrollo medio (Argentina y Uruguay) en los procesos de institucionalización del monitoreo y la evaluación, se evidencian brechas entre las diversas áreas o sectores, los cuales -además de trabajar con lógicas fragmentadas- presentan capacidades diferenciadas en cuanto a sus prácticas de sistematización de acuerdo a los sectores a los que pertenecen. Por ejemplo, en Argentina las áreas de educación en general han mostrado un mayor desarrollo de prácticas evaluativas que las áreas de desarrollo social. Por otro lado, en el caso de México, se plantea que la mayor parte de sus capacidades están centradas en las áreas vinculadas al desarrollo social (en el CONEVAL y la Secretaría de Desarrollo Social) mientras que la capacidad de áreas no sociales en materia de monitoreo y evaluaciones es más limitada. Se plantean tres síntomas de esta brecha de capacidades: la proliferación de indicadores que no son usados, problemas en la calidad de algunas de las evaluaciones y la casi total falta de evaluaciones a nivel de los gobiernos subnacionales (Arizti y Fernando Castro 2010).

En aquellas áreas donde el monitoreo y la evaluación presenta un menor desarrollo institucional, se identifica la escasa sistematización de "lo que el Estado hace" y esto se relaciona con que la práctica de registro y de sistematización durante la implementación de una política puede ser considerada como un trabajo con poco sentido. Este recelo tiene asidero en numerosas ocasiones cuando los esfuerzos de sistematización quedan archivados o, si llegan a manos de los decisores públicos, están lejos de influir o generar algún aporte a la política pública evaluada. A la par, esas brechas de capacidad entre sectores también se evidencian en los sistemas de información que se utilizan en cada área. La capacidad de registro y la calidad de los sistemas de información están íntimamente relacionados: es posible tener los mejores sistemas pero que resulten inútiles sin la producción de información interna y registros por parte de las áreas del Estado y, a la inversa, además de producir información se hace necesario que ésta se encuentre ordenada por medio de sistemas que sean instrumentos útiles y confiables para la toma de decisiones. Otra causa de fondo se vincula a que la sistematización de información en general se requiere desde una exigencia asociada a la necesidad de control y esto genera un escenario de aversión y de resistencia. El desafio se centra en encontrar modalidades de trabajo que conviertan la práctica de sistematización en una herramienta útil y en encontrar incentivos que generen compromisos en los diversos niveles y que conjuguen voluntades tanto a nivel técnico como político para favorecer el registro y la sistematización continua al interior del propio gobierno. 
Para analizar las brechas de capacidades - además de las referidas al registro y la calidad de los sistemas de información- es posible basarse en evidencia al considerar las características de las propias áreas de monitoreo y evaluación; si es que existen: cuáles son sus objetivos, funciones, la cantidad y el nivel de profesionalización de su personal. Las agencias de monitoreo y evaluación más desarrolladas institucionalmente -como el CONEVAL de México- se alejan de la visión de mero control y plantean la preocupación por institucionalizar su ejercicio en las diversas áreas para hacer sentir que se trata de un instrumento que da frutos y de diferentes estilos.

Las instituciones clave para el monitoreo y la evaluación encuentran el desafio de que en su propio diseño consoliden un tipo de institucionalidad pública que sea consistente con un enfoque de derechos. Esto se torna central en el marco de una tendencia que ha cobrado relevancia en el último tiempo referida a la incorporación y/o consolidación del enfoque de derechos humanos en el campo de las políticas públicas en general. En este sentido, ha habido cierto consenso en que es necesario repensar las políticas públicas como parte de las obligaciones del Estado para el cumplimiento efectivo de derechos asociados a la existencia de una ciudadanía social, lo que significa la priorización de los principios de exigibilidad, integralidad y universalidad (Cunill Grau 2010). En materia de monitoreo y evaluación, la incorporación del enfoque de derechos aún no ha llegado a permear en la administración pública y su inclusión plantea una serie de desafios adicionales. Al incorporar el principio de igualdad y universalidad, este enfoque coloca en el centro de las preocupaciones el modo como la intervención evaluada aborda las situaciones de desigualdad del contexto, qué construcción de sentido promueve en sus acciones y los potenciales efectos en los esquemas de organización familiar y en los roles de varones y mujeres y; a partir del principio de exigibilidad, enfatiza en valorar los mecanismos de reclamo y garantía de forma que sean insumos para el monitoreo y la evaluación de políticas conforme a derechos (IPPDH 2014).

Cabe considerar una mención particular al principio de integralidad que incorpora el enfoque de derechos. Este se entiende a partir del reconocimiento de la interdependencia entre los derechos económicos, sociales y culturales y de los derechos políticos y civiles (Cunill Grau 2010). En este marco, las políticas serían integrales cuando demandan coordinar aproximaciones multidisciplinarias, transversales, capaces de reconocer el cambiante escenario entre la especificidad de una causa y su papel en el conjunto de determinantes intervinientes en una problemática dada (Acuña 2010). En materia de monitoreo y evaluación, algunos autores plantean que ante la necesidad de medición/ observación cuantificable, la tendencia hacia la evaluación por resultados -aunque de por sí valiosa- muchas veces se ha planteado de manera "angosta"; acotada a una unidad de gestión, sin ponderar los efectos sistémicos que hacen a la coordinación/integralidad del accionar entre sectores, jurisdicciones y organismos públicos y, en ocasiones, tendiendo a obturar el reconocimiento de lo que falta cubrir con el accionar estatal, lo 
ausente por caer en los intersticios entre programas y políticas (Acuña 2010). Los sistemas de monitoreo y evaluación en funcionamiento, en general, requieren la generación de cambios que permitan adoptar una racionalidad integradora acorde a los desafios de integralidad en tanto continúan centrándose en criterios de valoración de la gestión pública por sectores (Cunill Grau; 2005).

En este sentido, un desafio central se basa en contar con sistemas de monitoreo y evaluación que puedan aportar y ser instrumentos que favorezcan la integralidad de las políticas públicas. En el marco de estas preocupaciones, se ha planteado en México la conformación de un sistema integral de monitoreo y evaluación basado en resultados conformado por la Secretaría de Hacienda en coordinación con la Secretaría de la Función Pública y el CONEVAL con el propósito de proveer a las dependencias y entidades responsables de los programas federales, información actualizada y periódica sobre la utilización de los recursos asignados, los avances y el logro de sus resultados (Secretaría de Hacienda y Crédito Público, Secretaría de la Función Pública y Consejo Nacional de Evaluación de la Política de Desarrollo Social 2007). Sin embargo, más allá de su postulación formal, este sistema no ha logrado consolidarse con un carácter integral aunque sí ha generado avances en compartir información y en experiencias de trabajo conjunto entre las tres áreas involucradas.

Entre los desafíos que la búsqueda de integralidad en el campo de la política social genera en el monitoreo y evaluación destaca la importancia de contar con sistemas de información para una gestión integral que vayan más allá de lo sectorial. Para que la información contribuya a la toma de decisiones relativas a propuestas integrales - que comportan la articulación de diversas intervenciones - se requieren sistemas de información integrados (Cecchini y Martínez 2011).

Otras brechas que se identifican y se convierten en retos para los sistemas de monitoreo y evaluación hacen referencia al establecimiento de indicadores que vayan más allá de lo sectorial y se orienten a la integralidad. Los indicadores en materia de derechos humanos pueden definirse como herramientas conceptuales destinadas a reflejar el estado de satisfacción de un derecho; a la vez, al proponer una mirada dinámica, enfatizar en la capacidad de reflejar los esfuerzos hechos por el Estado para satisfacer plenamente esos derechos (Courtis 2010). En resumen, para que la información contribuya a la toma de decisiones relativas a propuestas integrales -que comportan la articulación de diversas intervenciones- se requieren sistemas de información con indicadores sociales, financieros y operacionales que no solo contemplen su gestión específica sino que también abarquen con mayor amplitud el campo de las políticas públicas colaborando en la construcción de un sentido integrado de las acciones -y omisiones- del Estado (Cecchini y Martínez 2011). 


\subsection{La utilización de las evaluaciones y la perspectiva de aprendizaje}

A pesar de avances importantes en la dimensión institucional de la función de monitoreo y evaluación, la utilización efectiva de la evaluación para la mejora, la rendición de cuentas y la planeación presupuestaria siguen siendo un objetivo elusivo de la política de evaluación (Maldonado 2014). Es decir, aunque la relevancia del monitoreo y evaluación sea cada vez más reconocida tanto en el campo académico como al interior de la gestión pública, en la práctica esta dupla no se ha instalado como instrumento significativo para la gestión de políticas públicas.

Esto se explica en parte debido a que el fomento del monitoreo y la evaluación ha sido liderado de modo principal por ministerios centrales de planeación y de hacienda, interesados en lograr transparencia con respecto a los efectos e impactos del gasto público; sin haber generado convicciones y compromisos por parte de los ministerios y áreas implementadoras (Mokate 2003). En este sentido, una preocupación fuertemente extendida e impulsada por el enfoque basado en resultados se vincula a la utilización de los hallazgos del monitoreo y la evaluación en la definición del presupuesto. Esto se reconoce como un desafio tanto en las experiencias de Colombia y México. Sin embargo, plantea importantes interrogantes: ¿están los sistemas de monitoreo y evaluación en condiciones para influir si quitar o asignar más presupuesto? ¿Qué tipo de hallazgos de las evaluaciones o recomendaciones deberían ser tomadas en cuenta en decisiones presupuestarias? Algunos autores sostienen que es dificil atar los resultados del monitoreo y la evaluación a conclusiones contundentes sobre si debe aumentarse o reducirse el presupuesto asignado a determinada política (Cejudo 2011). Es claro que la decisión mecánica de quitar presupuesto a un programa porque no funciona de forma eficiente se enfrentaría a importantes riesgos asociados a la garantía efectiva de ciertos derechos. Sobre todo al considerar el principio que impone el enfoque de derechos relativo a garantizar el máximo de recursos disponibles para efectivizar progresivamente el pleno ejercicio de los derechos sociales (IPPDH 2014).

Según Hallam y Bonino (2013) es posible identificar diversos usos de la evaluación: el uso directo o instrumental por medio de las cuales se recogen los resultados, hallazgos, y recomendaciones; el uso conceptual que hace referencia a cómo varias evaluaciones combinadas con otras evidencias y opiniones logran documentar los debates e influir en la toma de decisiones; uso del proceso cuando se ofrecen oportunidades para intercambiar información y aprender del proceso de la evaluación y, finalmente, el uso simbólico para justificar las preferencias y acciones preexistentes o reforzar la confianza de aquellos que quieren presionar para lograr el cambio.

Una causa que explica por qué el uso directo o instrumental de las evaluaciones es poco frecuente refiere a la ausencia de mecanismos o canales para conectar los resultados de las evaluaciones estrechamente con el proceso de hechura de políticas; incluso cuando 
esos mecanismos se establecen hacen falta incentivos para mantenerlos y mejorar su funcionamiento (Weiss; 1999).

Los enfoques de la evaluación muy centrados en la rendición de cuentas, la exigencia por parte de un donante o la búsqueda de control desde los altos mandos se detectan como factores que influyen fuertemente en su escasa utilización ya que los participantes tienden a sentirse juzgados y con menos voluntad de participar en los procesos de evaluación, y posteriormente, de integrar hallazgos y las recomendaciones resultantes (Hallam y Bonino; 2013).

El escaso uso de las evaluaciones se basa en que las perspectivas predominantes han estado basadas en el control ya sea del gasto, "desde arriba" (de los gerentes públicos) o desde la ciudadanía; mientras que la función referida al aprendizaje no ha recibido suficiente atención en la práctica. Se hace necesario encontrar mecanismos e incentivos para que los sistemas de monitoreo y evaluación tiendan a incorporar mejoras durante la implementación, generar procesos de retroalimentación permanente, fortalecer capacidades al interior del estado y funcionar como mecanismo integrador de la política pública. En esta línea, Ridell (2009) sostiene:

Si se viera a las evaluaciones menos como una amenaza y más como una «herramienta de aprendizaje» para ayudar a los gestores a desplegar sus recursos de una manera más eficaz, entonces, por un lado, los evaluadores se sentirían más motivados para escribir evaluaciones menos cautelosas e informes más útiles, y por otro lado, habría más posibilidades de que los gestores se tomen más interés en comprometerse más profundamente con las recomendaciones presentadas y con su seguimiento (apud Hallam y Bonino 2013: 49).

En este marco, resulta relevante considerar las funciones que se han reconocido en los sistemas de monitoreo y evaluación. De acuerdo a cómo se las ha estudiado en los diagnósticos de los diversos países latinoamericanos, en el marco del programa de fortalecimiento de los sistemas de monitoreo y evaluación (M\&E), ${ }^{8}$ se pueden identificar cuatro grandes categorías de funciones (Canievsky 2007): a) planificación gubernamental (global o sectorial); b) la asignación del gasto; c) el aprendizaje; d) la rendición de cuentas dentro y fuera de la administración pública.

Las funciones predominantes en los sistemas de monitoreo y evaluación guardan relación con los intereses básicos de las iniciativas de reforma más amplias de las que formó parte, y con el entorno político e institucional en el que se desarrolle (Villareal 2007). La utilización que se realiza de la evaluación es fuertemente influenciada, además, por concepciones y perspectivas subyacentes. 
Cabe destacar que tanto la asignación del gasto como la rendición de cuentas están sesgadas hacia una visión del control mientras que la planificación gubernamental y manifiestamente el aprendizaje para mejorar la gestión se ligan más estrechamente a la perspectiva de aprendizaje.

La visión del control lleva a considerar al miedo como un elemento positivo que colabora en generar altos niveles de logro del monitoreo y evaluación. Orrego y Traverso (2013) sostienen que el caso chileno es usualmente destacado como una experiencia exitosa de implantación de un sistema de monitoreo y evaluación, y respecto a esto, señalan la importancia del Ministerio de Hacienda, por sus altos niveles de capacidad, de respeto y de miedo que genera en el resto de la administración pública, lo que ayudaría a que el monitoreo y la evaluación tengan altos niveles de logro.

En contraste, otras visiones plantean que un desafio importante para la política social es justamente poder convertir en aliado a las herramientas de MyE, a fin de generar procesos de aprendizaje que permitan mejorar su calidad. Cuando la visión de control se torna exclusiva o extremadamente predominante, es más probable que el monitoreo y la evaluación sean percibidos como una amenaza.

\section{Consideraciones finales: brechas por cerrar}

El presente trabajo ha buscado profundizar sobre las brechas por cerrar en materia de monitoreo y evaluación, tanto en el campo teórico como en las perspectivas subyacentes y en la práctica de la gestión pública. Se trata de distintos tipos de brechas, entendidas como distancias entre la situación actual y las aspiraciones o expectativas. Éstas se pueden reconocer en el ámbito del conocimiento teórico cuando se confunden las nociones de monitoreo y evaluación e incluso, en el otro extremo, cuando se sobreestiman sus distinciones planteando oposiciones -y extendiendo las brechas- en vez de entender ambos componentes como parte un proceso continuo, relacional y complementario. Sobre todo, se identifican brechas de capacidades que se reconocen en los desiguales niveles de institucionalización de los sistemas de monitoreo y evaluación entre países de la región; en las capacidades diferenciadas entre sectores o áreas de gobierno (desarrollo social, educación, salud, etc.) en sus sistemas de información y en las características de las propias áreas de monitoreo y evaluación; y también en la distancia entre el desarrollo institucional del monitoreo y evaluación en los diversos niveles de gobierno (en particular en el bajo nivel de desarrollo de los gobiernos subnacionales en comparación a los gobiernos nacionales). A la vez, se visualizan brechas en los sistemas de monitoreo y evaluación asociadas a las perspectivas subyacentes: entre el reconocimiento de derechos en la retórica, el logro progresivo de los mismos y su garantía efectiva; entre los procesos de institucionalización de la evaluación y la efectiva utilización de estos instrumentos y, a la vez, entre la utilización y la perspectiva de control. El desafio pareciera ser ir ce- 
rrando brechas, complementando visiones y construir un "entre" que tienda puentes a partir del aprendizaje.

La perspectiva de control presenta mayores pruebas de haber logrado que el monitoreo y la evaluación se tengan en cuenta en la gestión pública, sin embargo, no hay evidencia que esto repercuta en su uso instrumental para la mejora de las políticas públicas. La función de aprendizaje no ha sido priorizada en los gobiernos latinoamericanos también porque requiere multiplicar esfuerzos para generar un proceso de apropiación del monitoreo y la evaluación como instrumentos con altas potencialidades para el propio implementador de las políticas públicas. El rol de los gestores ha sido menospreciado en este marco cuando en realidad constituyen actores claves para favorecer la utilización de la información. En este sentido, el monitoreo o seguimiento puede resultar un proceso clave para incorporar cambios o ajustes oportunos durante la misma implementación y la evaluación permite profundizar en hallazgos y recomendaciones para mejorar aspectos concretos de las políticas públicas que requieran una aproximación adicional. Si se prioriza la perspectiva de aprendizaje, tanto el monitoreo como la evaluación presentan amplias potencialidades para generar procesos de apropiación por los propios implementadores de las políticas y, desde allí, generar aprendizajes conjuntos y procesos de integración.

En este marco, se propone adoptar una perspectiva sintetizadora, la cual utiliza elementos del enfoque racionalista y del enfoque interpretativo. Del primero, se extrae la consideración del monitoreo y la evaluación como una herramienta dirigida a promover el aprendizaje a través de la construcción de un conocimiento sobre la realidad de las políticas que tenga validez intersubjetiva y, en relación al segundo, se enfatiza la necesidad de reconocer la naturaleza plural de esa realidad, la coexistencia de sentidos cuyos contenidos y atributos deberán ser indagados y la inevitable movilización de valores por parte del evaluador, lo que lo convierte en un constructor de argumentaciones con incidencia sobre los procesos sociales y decisorios que se dan al interior de la organización evaluada (Martínez Nogueira 2013). De este modo, al reconocer la existencia de diversos actores en el proceso evaluativo, se consideran las múltiples racionalidades e intereses en juego. Así, además de valorar el rol de los propios implementadores, las evaluaciones deben involucrar a las personas y a las comunidades. La incorporación de líderes políticos que toman decisiones, de los investigadores profesionales y de la comunidad local al proceso de investigación representa una alternativa válida para asegurar una retroalimentación oportuna y democratizar el proceso de producción y utilización del conocimiento, lo cual implica, la necesidad de promover una investigación comprometida o militante que integre: la revalorización del conocimiento popular, la desmitificación del método científico y la participación genuina de la gente en el proceso de investigación y acción (Peterson; 1992). 
Finalmente, es necesario recalcar que en este marco el monitoreo y la evaluación constituyen principalmente un proceso de transformación; el monitoreo de un programa en funcionamiento o la evaluación de la factibilidad de realización de otro programa implican pensar en un cambio en la forma en la que se está operando; se espera que resultados de la evaluación brinden elementos para generar algún tipo de modificación en la estrategia o metodología de intervención implementada, priorizando el aprendizaje (Niremberg et al; 2007). El desafio se centra en que el monitoreo y la evaluación repercutan en las políticas públicas para que éstas generen transformaciones positivas de la realidad y mejoren las condiciones de vida de las personas.

\footnotetext{
${ }^{1}$ El desarrollo del presente trabajo y los ejemplos que se exponen sobre los casos de México y Colombia toman como insumo información recolectada y reflexiones realizadas en el marco de una beca de estancia de investigación que la autora realizó en el Centro Regional para el Aprendizaje en Evaluación y Resultados (CLEAR) para América Latina (México).

${ }^{2}$ Algunos de los autores de referencia en la región que acuden a esta definición son García López y García Moreno (2010), Pallaveccini (2014) y Mokate (2003).

${ }^{3}$ (Del ingl. monitor):1. m. Aparato que revela la presencia de las radiaciones y da una idea más o menos precisa de su intensidad. Suelen ser detectores muy sensibles y de poca precisión; 2 . m. Aparato receptor que toma las imágenes directamente de las instalaciones filmadoras y sirve para controlar la transmisión. Monitor, ra. (Del lat. monĭtor, -ōris):1. m. y f. Persona que guía el aprendizaje deportivo, cultural, etc. 2. m. Hombre que amonesta o avisa; 3 . m. En el Ejército, ayudante de los profesores de educación física; 4. m. Subalterno que acompañaba en el foro al orador romano, para recordarle y presentarle los documentos y objetos de que debía servirse en su peroración; $5 . \mathrm{m}$. Esclavo que acompañaba a su señor en las calles para recordarle los nombres de las personas a quienes iba encontrando (Real Academia Española, 2012).

${ }^{4}$ En función de la palabra monitor, se reconoce en el diccionario panhispánico de dudas (DPD) de la Real Academia, la existencia de los verbos "monitorear/monitorizar" (la primera de uso más extensivo en América y la segunda en España) pero su significado es asociado exclusivamente a su origen inglés (dispositivo o pantalla de control) con el sentido de 'vigilar o seguir [algo] mediante un monitor (Real Academia Española, 2012). ${ }^{5}$ A lo largo del documento se utilizarán como sinónimos y de forma alternativa las palabras monitoreo y seguimiento.

${ }^{6}$ La gestión basada en resultados propone cambiar desde perspectivas de sistemas de control centradas en la rigurosidad de los procedimientos hacia un enfoque basado en resultados hacia el servicio y bienestar (CLAD y BID, 2007).

${ }^{7}$ Esta consideración se basa en el análisis de García López y García Moreno (2010) que analizan - a partir de un modelo conceptual que incluye el monitoreo y evaluación como uno de sus pilares - el nivel de los países latinoamericanos en materia de gestión basada en resultados para el desarrollo.

${ }^{8}$ El proyecto "Fortalecimiento de los sistemas de monitoreo y evaluación en América Latina y el Caribe" es una iniciativa del Banco Mundial y el Consejo Latinoamericano de Administración Pública (CLAD) que ha estudiado, en una primera etapa, los sistemas nacionales de M\&E de diversos países de la región con una base teórico metodológica común (Canievsky, 2007).
} 


\section{Referencias bibliográficas}

Acuña, Carlos (2010) Los desafíos de la Coordinación y la Integralidad para las Políticas y la Gestión Pública en América Latina. Una Introducción. http://www.jefatura.gob.ar/archivos/pme/publicaciones/desafios_coordinacion.pdf Accesado el 30 de Septiembre 2014.

Aguilar Villanueva, Luis (1992) "Estudio Introductorio”. En AguilarVillanueva, Luis (comp.) El estudio de las políticas. México: Miguel Ángel Porrúa:2 - 29.

Arizti, Pedro y Fernando Castro, Manuel (eds.) (2010) Mejorando la calidad del gasto público a través del uso de información de desempeño en México. Washington D.C: Grupo Banco Mundial Editores.

Asamblea Constituyente Nacional (1991) Constitución Política de Colombia. http:// www.alcaldiabogota.gov.co/sisjur/normas/Norma1.jsp?i=4125, Art. 343 y 344

Accesado el 19 de octubre 2014.

Canievsky, Carlos (2007) Diagnóstico de los sistemas gubernamentales de monitoreo y evaluación en Argentina. http://siare.clad.org/siare/innotend/evaluacion/argentina.pdf Accesado el 23 de septiembre 2014."

Cecchini, Simone y Martínez, Rodrigo (2011) Protección Social Inclusiva en América Latina. Una mirada integral, un enfoque de derechos. Santiago de Chile: CEPAL.

Cejudo, Guillermo (2011) "Sobre el Uso de las Evaluaciones”. En Cejudo, Guillermo y Maldonado Claudia (eds.) De las Recomendaciones a las Acciones: La Experiencia del Premio 2011 Programas Federales Comprometidos con el Proceso de Evaluación. México: Secretaría de la Función Pública-CLEAR-CIDE: 11-28.

CLAD y BID (2007) Modelo abierto de gestión para resultados en el sector público. Nueva York - Caracas: CLAD y BID.

Courtis, Christian (2010) “Apuntes sobre la elaboración y utilización de indicadores en materia de derechos económicos, sociales y culturales”. En Arcidiácono, Pilar y otros (coord.) Derechos sociales: justicia, política y economía en América Latina. Bogotá: Siglo de Hombre Editores y LAEHR: 389- 427.

Cunill Grau, Nuria (2005) La intersectorialidad en el gobierno y gestión de la política social. Santiago de Chile: CLAD. 
Cunill Grau, Nuria (2010) "Las políticas con enfoque de derechos y su incidencia en la institucionalidad pública". Revista del CLAD Reforma y Democracia No. 46. http://old.clad.org/portal/publicaciones-del-clad/revista-clad-reforma-democracia/ articulos/046-febrero-2010/cunill Accesado el 03 de febrero 2015

Cunill Grau, Nuria, y Ospina, Sonia (2007) Fortalecimiento de los sistemas de monitoreo y evaluación (MEE) en América Latina. Washington, D.C - Caracas: Proyecto CLADBanco Mundial.

Di Virgilio, María Mercedes y Solano, Romina (2013) Monitoreo y evaluación de políticas, programas $y$ proyectos sociales. Buenos Aires: CIPPEC y UNICEF.

Feinstein, Osvaldo (2010) "Relaciones entre la evaluación de las políticas y la calidad de los servicios". Revista del CLAD Reforma y Democracia No. 46: http://old.clad.org/ portal/publicaciones-del-clad/revista-clad-reforma-democracia/articulos/046-febrero-2010/feinstein Accesado el 03 de febrero 2015.

Feinstein, Osvaldo (2012) "La institucionalización de la evaluación de políticas públicas en América Latina". Presupuesto y Gasto Público 68: 41-52.

García López, Roberto y García Moreno, Mauricio (2010), Managing for development results. Progress and challenges in Latin America and the Caribbean. Washington, DC, United States: Inter-American Development Bank.

Guzmán, Marcela (2007) "Evaluación de Programas. Notas Técnicas”. Serie Manuales ILPES 64: 1 - 37.

Hallam, Alistairy y Bonino, Francesca (2013) Usando evaluaciones para un cambio real: Perspectiva de los especialistas humanitarios. Londres: ALNAP/ODI.

\section{IPPDH - Instituto de Políticas Públicas en Derechos Humanos MERCOSUR}

(2014) Ganar derechos. Guía para la formulación de políticas públicas basadas en derechos. Versión preliminar (inédito).

Maldonado Trujillo, Claudia (2014) “¿Evaluación para qué? Reflexiones en torno a la utilización de las evaluaciones.” (Inédito).

Martínez Nogueira, Roberto (2013) Clase 7: La evaluación de las políticas públicas. Argentina: Maestría en Desarrollo Humano, FLACSO (Inédito). 
Mokate, Karen (2003) Convirtiendo el "monstruo" en aliado: la evaluación como herramienta de la gerencia social. Washington: Instituto Interamericano para el Desarrollo Social - Banco Interamericano de Desarrollo.

Nirenberg Olga, Brawerman Josette, y Ruiz Violeta (2003) Programación y evaluación de proyectos sociales. Buenos Aires: Paidós.

Nirenberg, Olga; Brawerman, Josette y Ruiz, Violeta (2007) Evaluar para la transformación Innovaciones en la evaluación de programas y proyectos sociales. Editorial. Buenos Aires: Grupo Editorial Planeta- Editorial Paidós.

OECD (2002) Glosario de los principales términos sobre evaluación y gestión basada en resultado. http://www.oecd.org/development/peer-reviews/2754804.pdf Accesado el 20 de octubre 2014.

Orrego, Ariel y Traverso, Diego (2013) “Monitoreo y evaluación de resultados a nivel subnacional en Chile: ¿En busca de la coordinación intergubernamental?, en Maldonado Claudia. y Galíndez, Cristina (ed.) Monitoreo, evaluación y gestión por resultados. Aprendizajes y cooperación sur - sur para la innovación: el papel de los actores subnacionales. México: CIDE - Centro Clear para América Latina:199 - 223.

Ospina, Sonia (2000) Evaluación de la gestión pública: conceptos y aplicaciones en el caso latinoamericano. Santo Domingo:V Congreso Internacional del CLAD sobre Reforma del Estado y la Administración Pública.

Pallavicini, Violeta (2014), "Evaluación de políticas públicas y Gestión por Resultados: el reto de las administraciones públicas “. En Ríos Cázares, Alejandra (coord.) La evaluación de políticas públicas en América Latina: métodos y propuestas docentes. México: CIDE-Centro CLEAR para América Latina-Red Interamericana de Educación en Administración Pública:49 - 63.

Pedersen, Duncan (1992) "El dilema de lo cuantitativo y lo cualitativo: de las encuestas a los métodos rápidos de investigación en salud”. En Lolas Stepke Fernando y col., Ciencias Sociales y Medicina, Perspectivas Latinoamericanas. Santiago de Chile: Ed. Universitaria201 - 211.

PNUD (2009) Manual planificación, seguimiento y evaluación de los resultados de desarrollo. New York: PNUD.

Real Academia Española (2012) Diccionario de la lengua española. http://www.rae.es/ Accesado el 15 de octubre 2014. 
Romero Jiménez, Máximo (2013) “Desafios y limitantes para la cooperación internacional mexicana en monitoreo y evaluación”. En Maldonado, Claudia y Galíndez, Cristina. (ed.) Monitoreo, evaluación y gestión por resultados. Aprendizajes y cooperación sur - sur para la innovación: el papel de los actores subnacionales. México: CIDE-Centro Clear para América Latina:31 - 39.

\section{Secretaria de Hacienda y Crédito Público; Secretaría de la Función Pública} y Consejo Nacional de Evaluación de la Política de Desarrollo Social (2007) Lineamientos generales para la evaluación de programas federales (LGEPF). http://www.coneval.gob.mx/rw/resource/coneval/eval_mon/361.pdf Accesado el 27 de julio 2014.

Subirats, Joan (1989) Análisis de políticas públicas y eficacia de la administración. Madrid: INAP.

Villareal Navarro, Julio (2007) Colombia y el Sistema de Monitoreo y Evaluación, SINERGIA. http://siare.clad.org/siare/innotend/evaluacion/colombia.pdf Accesado el 25 de septiembre 2014.

Vinocur, Pablo y Ruiz, Violeta (2013) Clase 3: Planificación y Evaluación de Políticas Públicas. Argentina: Maestría en Desarrollo Humano, FLACSO (Inédito).

Weiss Hirschon, Carol (1999) The Interface between Evaluation and Public Policy. London, Thousand Oaks and New Delhi: SAGE Publications.

Zaltsman, Ariel (2006) Desarrollo de la Capacidad de Evaluación: Experiencia con la institucionalización de sistemas de monitoreo y evaluación en cinco países latinoamericanos: Argentina, Chile, Colombia, Costa Rica y Uruguay. Washington: Banco Mundial. 